\title{
Rapid Generation of Complex Molecular Architectures by a Cata- lytic Enantioselective Dearomatization Strategy
}

Alice E. Williamson

Tifelle Ngouansavanh

Robert D. M. Pace

Anna E. Allen

James D. Cuthbertson

Matthew J. Gaunt*

Synlett 2015, 26, 116.

Due to a production error, in Table 2, fourth example, the amendments were added to the text, rather than replacing it. This mistake was introduced in house and has been corrected in all versions. 